\title{
Sistematización del programa de Teledermatología de la Universidad de Caldas - departamento de Caldas, 2002- 2010
}

\author{
Ana Patricia Insuasty'; Felipe Jaramillo²; José Arnoby Chacón ${ }^{3}$
}

\section{RESUMEN}

Introducción: el uso de la teledermatología como herramienta diagnóstica está introduciéndose en la práctica diaria del dermatólogo. Múltiples factores explican el gran potencial que la teledermatología tiene en un país como el nuestro. Al momento, existen pocos estudios publicados acerca del tema y ninguno de ellos describe la aplicación y el impacto que generan los programas de teledermatología en nuestro medio.

Materiales y métodos: estudio observacional-retrospectivo, realizado en el programa de Teledermatología de la Universidad de Caldas entre 2002 y 2012. Se describieron las características sociodemográficas, la frecuencia de enfermedades, la concordancia entre los diagnósticos emitidos por el médico general y el dermatólogo, la capacidad resolutiva, los motivos de derivación a la consulta presencial y los problemas para emitir un diagnóstico.

Resultados: se analizaron 4333 teleconsultas y 4713 diagnósticos. Los grupos de enfermedades más frecuentes en la teleconsulta fueron las enfermedades inflamatorias (49\%), seguidos por las neoplasias benignas (19\%). La concordancia diagnóstica fue del 52,4\%. La capacidad resolutiva fue del 68,5\%. El motivo de derivación más frecuente fue la confirmación diagnóstica. El 2,2\% de los casos presentó dificultades en la teleconsulta. El principal motivo fueron los problemas con las imágenes fotográficas.

Conclusión: las características sociodemográficas y las enfermedades consultadas presentan una distribución similar a la de otros estudios. La capacidad resolutiva es alta y evita más de la mitad de las derivaciones a la consulta presencial. En pocos casos se tuvieron inconvenientes para realizar la teleconsulta. El grado de concordancia con el diagnóstico del médico general es adecuado.

PALABRAS CLAVE: Telemedicina; Teledermatología; Dermatología; Colombia.

\footnotetext{
1. Médica dermatóloga, Universidad de Caldas, Manizales, Colombia. ORCID https://orcid.org/oooo-ooo1-5153-9530

2. Médico dermatólogo, dermatopatólogo. Coordinador, posgrado de Dermatología, Universidad de Caldas, Manizales, Colombia. ORCID https://orcid.org/oooo-ooo2-0982-4335

3. Médico, MSP. Profesor asociado, Universidad de Caldas, Manizales, Colombia. ORCID https://orcid.org/oooo-0001-6206-653x
}

Correspondencia: Ana Patricia Insuasty; email: insuasty87@gmail.com

Recibido: $12 / 03 / 20$; aceptado: 08/05/20

Cómo citar: Insuasty, AP; Jaramillo, F; Chacón JA. Sistematización del programa de Teledermatología de la Universidad de Caldas departamento de Caldas, 2002- 2010. Rev Asoc Colomb Dermatol. Vol 28(3): julio - septiembre, 2020, 226-239.

DOI: https://doi.org/10.29176/2590843X.1532

Financiación: ninguna, conflictos de interés: ninguno 


\section{SYSTEMATIZATION OF THE TELEDERMATOLOGY PROGRAM AT THE UNIVERSIDAD DE CALDAS - DEPARTMENT OF CALDAS, 2002-2010}

\section{SUMMARY}

Introduction: Teledermatology has been introduced as a novel diagnostic tool for the daily dermatology practice. Several aspects explain why teledermatology would have a huge potential in a country like our own. Up to this date, a very few studies about the subject, and none of them quantify the field impact of large-scale teledermatology programs in Colombian population.

Material and methods: This was a retrospective observational study, accomplished within the program of teledermatology of the Universidad de Caldas, between 2002 and 2012. The sociodemographic features, frequency of disease, diagnosis agreement between to the general physician and dermatologist, resolution capacity, reasons for referral to face-to-face consultation and the difficulties present while making a diagnostic were studied.

Results: A sample of 4333 consults with 4713 dermatologic diagnoses were analyzed. The inflammatory diseases were the most frequent diagnosis group (49\%), followed by benign neoplasms (19\%). The percentage aggregate agreement between diagnoses was 52.4\%. The resolution capacity was achieved of $66 \%$. The main reason for a referral was the need diagnostic confirmation. In some cases $(2.2 \%)$, due to low quality imaging, the dermatologists have had difficulties while providing with a diagnostic.

Conclusions: The distribution of the sociodemographic characteristics and dermatological diseases is similar to the ones found in previous studies. The resolution capacity achieved with teledermatology is representative enough to avoid almost half of the referrals to the face-to-face consultation. In few cases, there was issues regarding the develop of the teleconsult. The degree of agreement obtained with the general physician diagnosis was acceptable.

KEY WORDS: Telemedicine; Teledermatology; Dermatology; Colombia.

\section{INTRODUCCIÓN}

La teledermatología es una rama de la telemedicina, que se define como la práctica de la dermatología a distancia ${ }^{(1)}$. Utiliza dos sistemas, uno diferido o de almacenaje y otro en tiempo real, siendo el sistema diferido el más factible en la práctica diaria y, por lo tanto, el más utilizado ${ }^{(2,3)}$. Actualmente es considerada la disciplina clínica mejor estudiada de la telemedicina y la de mayor influencia potencial en decisiones de política sanitaria $^{(4)}$.

La dermatología es una especialidad amplia que puede presentar enfermedades que le son propias o reflejar las de otros órganos y constituye uno de los principales motivos de consulta para el médico general (5). En nuestro país se encuentra en el tercer nivel de atención en salud; muchas personas no cuentan con la posibilidad de acceder a los servicios dermatológicos por múltiples factores, que incluyen: geográficos, políticos, económicos, de movilidad, encarcelamiento y de marginalización social. Además, los especialistas en dermatología se encuentran distribuidos en las principales ciudades del país, como Bogotá y Medellín, en el $30,6 \%$ y el 19,4\%, respectivamente, y el resto está distribuido en ciudades intermedias ${ }^{(6)}$, lo que limita más el acceso de la población rural a la especialidad; por lo tanto, surge la importancia de buscar alternativas para mejorar la inasequibilidad.

En Colombia, desde el 2006 se diseñaron políticas públicas para el ejercicio de la telemedicina, con la Resolución 2003 de $2014^{(7)}$ y con el apoyo de las tecnologías de la información y las comunicaciones o TIC ${ }^{(8)}$ buscan prestar y garantizar el acceso a los servicios de salud en las zonas apartadas de la geografía nacional a través de la telemedicina. En un estudio exploratorio realizado en el 2014, los autores encontraron que, de los 748 prestadores de servicios de salud encuestados, el $15,5 \%$ refirió prestar alguna actividad en telemedicina, y de estos, solo el 15,8\% en teledermatología ${ }^{(9)}$.

El programa de Teledermatología fue pionero del proyecto de telesalud ${ }^{(10)}$ de la Universidad de Caldas. 
Funcionó en el posgrado de Dermatología con la modalidad de teleconsulta diferida, en el período comprendido entre noviembre de 2002 y julio de 2012. Las teleconsultas realizadas procedían de servicios de salud de atención primaria de varios municipios del departamento de Caldas y de algunos municipios de otros departamentos del país. El equipo de telesalud conformado principalmente por un médico líder e ingenieros de sistemas viajaba a los diferentes municipios para instalar equipos y capacitar al médico general en el manejo de la plataforma. El médico también fue capacitado en semiología, técnicas de biopsias y en la toma de fotografías médicas representativas de la lesión y de la enfermedad. El médico general, previo consentimiento informado, evaluaba al paciente, llenaba una ficha clínica preformada y adjuntaba una o varias imágenes fotográficas en la plataforma. En un segundo tiempo, mediante la misma plataforma, un dermatólogo, quien es el segundo autor de este estudio (FJA), en compañía de residentes y estudiantes analizó y definió la conducta de todas las teleconsultas. Cuando se requería una biopsia, el médico general recibió instrucciones: sobre la foto clínica del paciente se dibujó el sitio y el tipo de biopsia que debía realizarse. El estudio de histología fue llevado a cabo en la Universidad de Caldas y el resultado fue evaluado mediante teleconsulta. La teleconsulta funcionó más como una teleinterconsulta, ya que el paciente, en la mayoría de los casos, fue manejado por el médico general con la teleorientación del dermatólogo.

En Colombia hay pocos estudios acerca de la teledermatología. En estos se evalúa la confiabilidad diagnóstica ${ }^{(11-13)}$. Ninguno de los estudios publicados realiza la descripción de los programas de Teledermatología que existen en el país. Esto crea la necesidad de conocer cuál es la experiencia nacional de estos programas, para aportar a la caracterización de los pacientes con enfermedades dermatológicas, además de mostrar los beneficios y las limitaciones que se puedan presentar en los pacientes, los médicos generales y los dermatólogos que acceden a la prestación de servicios de salud a través de la telemedicina en nuestro medio.

El objetivo general de este estudio fue describir las características de las teleconsultas de los municipios del departamento de Caldas, realizadas durante el funcionamiento del programa de Teledermatología de la Universidad de Caldas.

\section{MATERIALES Y MÉTODOS}

Se llevó a cabo un estudio observacional-retrospectivo de las teleconsultas realizadas en pacientes de todas las edades en el programa de Teledermatología de la Universidad de Caldas, desde noviembre de 2002 hasta junio de 2012. Se incluyeron las teleconsultas realizadas en el departamento de Caldas y se excluyeron las teleconsultas utilizadas como prueba piloto al inicio del programa, las teleconsultas de control y las teleconsultas con información insuficiente.

Se elaboró un base de datos para ingresar la información recolectada. Las variables estudiadas fueron edad, sexo, lugar de procedencia, fototipo de Fitzpatrick, diagnóstico realizado por el médico general, diagnóstico realizado por el dermatólogo, concordancia entre los diagnósticos del médico general y del dermatólogo, capacidad de resolución y motivos de valoración presencial.

Para la descripción de los diagnósticos se consideraron cinco grupos de enfermedades: infecciosas, inflamatorias, neoplasias benignas, neoplasias malignas y otras enfermedades; a su vez, estas categorías se clasificaron en subgrupos para facilitar la organización de los diagnósticos y poder obtener un mayor número de casos en cada subgrupo. En el grupo de neoplasias malignas se decidió no subagrupar, ya que los diagnósticos fueron pocos y cada uno tenía un número representativo de casos. Se consideró como diagnóstico 1 el diagnóstico realizado por el dermatólogo como motivo principal de teleconsulta, y diagnóstico 2 en el caso en que durante la teleconsulta se encontrara otro diagnóstico no relacionado con el anterior.

Se evaluó la concordancia entre los diagnósticos del médico general y el dermatólogo. Se utilizaron los datos clasificados en la categoría diagnóstico 1 (motivo principal de la teleconsulta) y se tomó como patrón de oro el diagnóstico realizado por el dermatólogo en la teleconsulta.

Se midió la concordancia de dos formas: 1. En relación con los diagnósticos propuestos por el médico general; y 2. En relación con estos diagnósticos, clasificados por grupos y subgrupos de las enfermedades. La segunda forma se utilizó para lograr una mayor uniformidad en los datos, debido a que el médico general utilizó una gran variabilidad de diagnósticos y de términos generales-inespecíficos tales como alopecia, dermatitis, cáncer de piel y nevo. 
Los criterios para definir la concordancia clasificada por los diagnósticos fueron: completa, cuando el diagnóstico emitido por el médico general fue igual al patrón de oro; parcial, cuando se emitían varios diagnósticos diferenciales o un término inespecífico aceptable que hacía referencia al patrón de oro; agregada, a la suma de la concordancia completa y parcial; y ninguna, cuando no coincidian ninguno de los diagnósticos.

Se definió como capacidad resolutiva el porcentaje de casos resueltos a través de la teleconsulta. Para la indicación dada por el dermatólogo se estableció el alta en caso de que el problema consultado se resolviera por medio de la teleconsulta, y derivación en caso de que fuese necesaria la valoración en la consulta presencial. Las razones para derivar a la consulta presencial incluyeron: necesidad de definir un diagnóstico, definir el tratamiento, procedimientos o consulta con otras especialidades.

Dentro de las dificultades que tuvo el dermatólogo al emitir un diagnóstico se incluyeron problemas con las imágenes o con los datos de la historia clínica.

Para el análisis estadístico se realizó el promedio y desviación estándar para la edad, proporciones para las variables categóricas. Para evaluar la concordancia realizada por los diagnósticos se realizaron proporciones, y para la concordancia por grupos y subgrupos de enfermedades, prueba de chi cuadrado e índice kappa. Las medidas se obtuvieron con el programa SPPS 21.

Este estudio fue presentado y aprobado con el acta n. ${ }^{\circ}$ 016 de 2016 por el comité de ética de la facultad de Medicina de la Universidad de Caldas. Se consideró una investigación sin riesgo, de acuerdo con la Resolución 8430 de 1993 del Ministerio de Salud.

\section{RESULTADOS}

Se recolectaron 5617 teleconsultas del programa de Teledermatología de la Universidad de Caldas realizadas entre noviembre de 2002 y junio de 2012, procedentes de diferentes centros de salud de 15 departamentos del territorio colombiano. Se descartaron 598 teleconsultas por pertenecer a otros departamentos, 137 teleconsultas que fueron utilizadas como prueba piloto, 166 teleconsultas por información insuficiente y 383 teleconsultas de control.
Finalmente se analizaron 4333 teleconsultas, 380 de estas tenían un segundo diagnóstico, para un total de 4713 diagnósticos analizados. En 412 teleconsultas el médico general no aportó ningún diagnóstico, por lo que para el análisis de concordancia diagnóstica se incluyeron 3921 teleconsultas.

Las teleconsultas pertenecieron a 24 municipios del departamento de Caldas realizadas durante el período comprendido entre noviembre de 2002 y marzo de 2010.

Las características sociodemográficas se muestran en la tabla 1. La edad media \pm desviación estándar de la población de estudio fue de $37 \pm 24,7$; el rango de edad de consulta más frecuente estuvo entre los 18 y los 45 años. El $62,7 \%$ de los pacientes fue de género femenino. Los tres fototipos más frecuentes de la población fueron III, II y IV, con $50 \%, 24 \%$ y $18,7 \%$, respectivamente. El 81,5\% de los pacientes pertenecieron al área urbana, siendo los municipios de La Dorada (47\%), Aguadas (19,8\%) y Salamina (7,2\%) los lugares que más casos aportaron al programa de Teledermatología.

El 28,6\% de la población perteneció al grupo pediátrico. En la tabla 2 se muestra la distribución del género y los grupos etarios, donde se observa un predominio del género femenino en ambos grupos.

El grupo de consulta más frecuente fue el de las enfermedades inflamatorias ( $\mathrm{n}=2310)$, seguido del grupo de las neoplasias benignas $(n=898)$ y del grupo de las enfermedades infecciosas $(n=736)$, como se observa en la figura 1.

En la figura 2 se comparan los grupos de enfermedades y los grupos etarios. El grupo más frecuente fue el de las enfermedades inflamatorias, tanto en la población pediátrica como en los adultos; el grupo de las enfermedades infecciosas predomina en la población pediátrica; y los grupos de las neoplasias benignas y malignas, en la población adulta.

En la tabla 3 se muestra la clasificación por grupos, el porcentaje de cada subgrupo y los tres diagnósticos más frecuentes en cada uno.

En la tabla 4 se muestra la concordancia con los diagnósticos clasificados en función de los grupos de las enfermedades. Mediante chi cuadrado se encuentra asociación estadísticamente significativa $(p=0,0001) \mathrm{y}$ un índice Kappa de o,7. 
Tabla 1. Características sociodemográficas

n

Edad

o a 17 años

18 a 45 años

46 a 60 años

$>61$

Género

Femenino

Masculino

Fototipo

I
III
IV
V
VI
Procedencia

$\begin{array}{lcc}\text { La Dorada } & 2038 & 47 \\ \text { Aguadas } & 859 & 19,8 \\ \text { Salamina } & 314 & 7,2 \\ \text { Pensilvania } & 196 & 4,5 \\ \text { Pácora } & 156 & 3,6 \\ \text { Riosucio } & 139 & 3,2 \\ \text { Supia } & 103 & 2,4 \\ \text { Otros municipios } & 528 & 12,2 \\ \text { Área } & & 81,5 \\ \text { Urbana } & 3530 & 18,5 \\ \text { Rural } & 803 & \end{array}$

$\%$ 28,6

32,2

17,5

21,7

$940 \quad 21,7$

$2716 \quad 62,7$

$1617 \quad 37,3$

$263 \quad 5,4$

1038

2171

$812 \quad 18,7$

$30 \quad 0,7$

$19 \quad 0,4$

0,4

4
0
7
7
4$$
, 4
$$

7




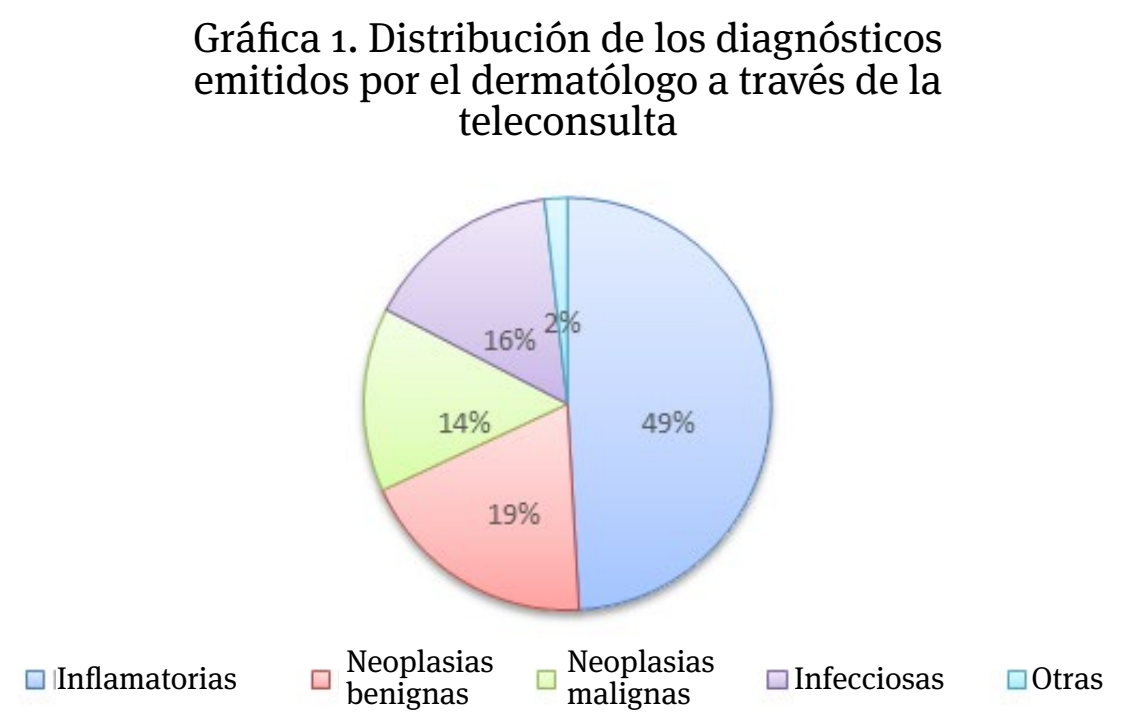

Figura 1. Distribución de los diagnósticos emitidos por el dermatólogo a través de la teleconsulta.

\section{Gráfica 2. Distribución de enfermedades por grupos etarios}

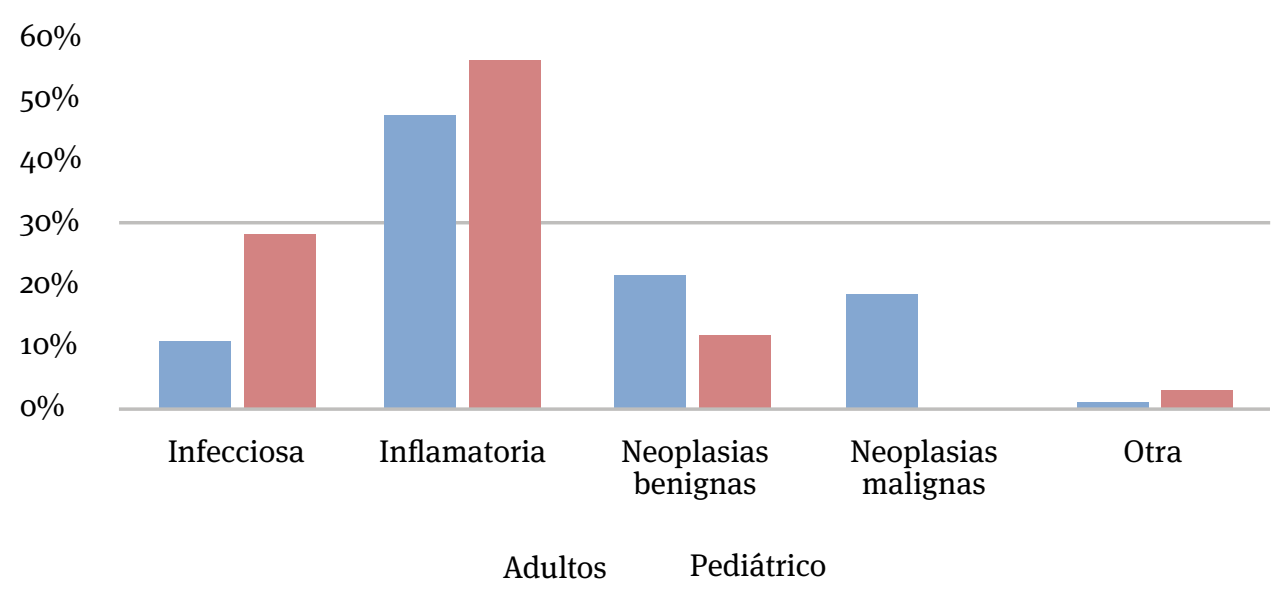

Figura 2. Distribución de enfermedades por grupos etarios 
Tabla 3. Grupos y subgrupos de enfermedades dermatológicas con los tres diagnósticos más frecuentes

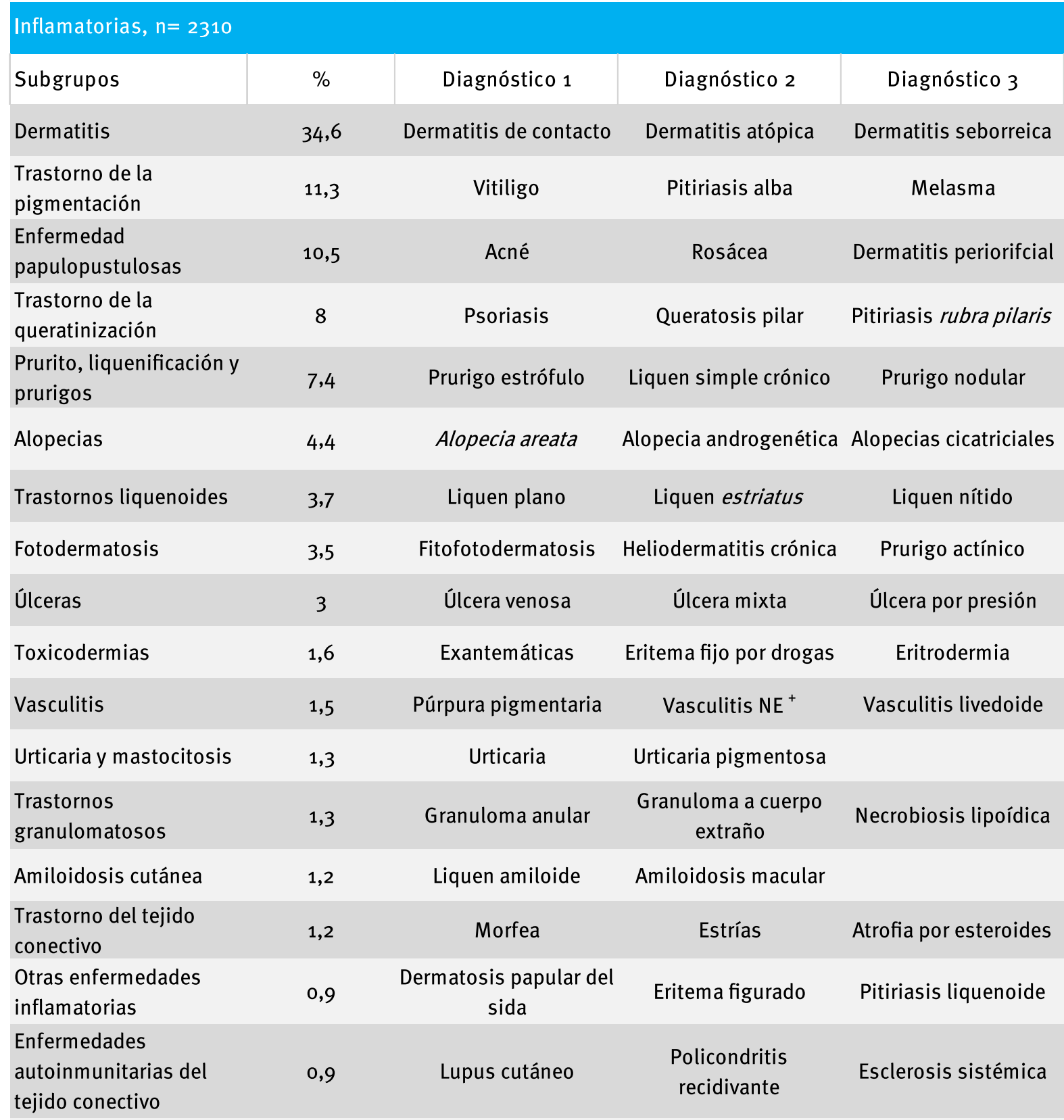

Trastornos de las glándul $\quad 0,6 \quad$ Miliaria Hiperhidrosis Hidradenitis supurativa

$\begin{array}{lcccc}\text { Trastornos inflamatorios } & 0,6 & \text { Balanitis simple } & \text { Glositis migratoria } & \text { Chalación } \\ \begin{array}{l}\text { Enfermedades } \\ \text { ampollosas }\end{array} & 0,3 & \text { Pénfigo foliáceo } & \text { Pénfigo vulgar } & \begin{array}{c}\text { Pénfigo benigno } \\ \text { familiar }\end{array} \\ \text { Dermatosis neutrofílicas } & 0,2 & \text { Síndrome de Sweet } & \text { Pioderma gangrenoso } & \\ \text { Paniculitis } & \mathbf{0 , 2} & \text { Eritema nodoso } & \text { Paniculitis NE } & \begin{array}{c}\text { Eritema indurado de } \\ \text { Bazin }\end{array}\end{array}$




\begin{tabular}{|c|c|c|c|c|}
\hline \multicolumn{5}{|c|}{ Neoplasias benignas, $n=898$} \\
\hline Subgrupos & $\%$ & Diagnóstico 1 & Diagnóstico 2 & Diagnóstico 3 \\
\hline Lesiones melanocíticas & 34,6 & $\begin{array}{l}\text { Nevo melanocítico } \\
\text { adquirido }\end{array}$ & Lentigo solar & $\begin{array}{l}\text { Nevo melanocítico } \\
\text { congénito }\end{array}$ \\
\hline Tumores epidérmicos & 22,3 & Queratosis seborreicas & $\begin{array}{c}\text { Dermatosis papulosa } \\
\text { nigra }\end{array}$ & \\
\hline $\begin{array}{l}\text { Hiperplasias y tumores } \\
\text { fibroblásticos }\end{array}$ & 13 & Queloide & Acrocordón & Dermatofibroma \\
\hline Lesiones vasculares & 10 & Hemangioma & Granuloma piógeno & Nevo rubí \\
\hline $\begin{array}{l}\text { Quistes } \\
\text { y pseudoquistes }\end{array}$ & 8,2 & Quiste infundibular & Mucocele & Quistes de milia \\
\hline Tumores de anexos & 5,7 & Tumor de anexos NE & Siringomas & Pilomatricoma \\
\hline Hamartomas & 3,2 & $\begin{array}{l}\text { Nevo epidérmico } \\
\text { verrucoso }\end{array}$ & Nevo sebáceo & Nevo de Becker \\
\hline $\begin{array}{l}\text { Otras neoplasias } \\
\text { benignas }\end{array}$ & 2,6 & Xantelasmas & $\begin{array}{l}\text { Xantogranuloma } \\
\text { juvenil }\end{array}$ & Lipoma \\
\hline \multicolumn{5}{|l|}{ Infecciosas, $n=736$} \\
\hline Subgrupos & $\%$ & Diagnóstico 1 & Diagnóstico 2 & Diagnóstico 3 \\
\hline Infecciones virales & 39,2 & Verruga viral & Molusco contagioso & Herpes zóster \\
\hline Infecciones micóticas & 27,3 & Pitiriasis versicolor & Tiña de los pies & Candidiasis \\
\hline $\begin{array}{l}\text { Infecciones } \\
\text { por parásitos }\end{array}$ & 13 & Escabiosis & Leishmaniasis & Larva cutánea migrans \\
\hline Infecciones bacterianas & 9,7 & Foliculitis & Impétigo contagioso & Forunculosis \\
\hline $\begin{array}{l}\text { Reacciones } \\
\text { exantemáticas por } \\
\text { infección }\end{array}$ & 6,6 & Pitiriasis rosada & Eritema multiforme & $\begin{array}{c}\text { Síndrome de Gianotti } \\
\text { Crosty }\end{array}$ \\
\hline $\begin{array}{l}\text { Infecciones por } \\
\text { micobacterias }\end{array}$ & 3 & Lepra & Lupus vulgar & Escrofuloderma \\
\hline Otras infecciones & 0,6 & $\begin{array}{c}\text { Síndrome verrucoso } \\
\text { tropical }\end{array}$ & $\begin{array}{c}\text { Síndrome ulcerativo } \\
\text { genital }\end{array}$ & \\
\hline \multicolumn{5}{|c|}{ Neoplasias malignas, $n=682$} \\
\hline & & Diagnóstico 1 & Diagnóstico 2 & Diagnóstico 3 \\
\hline & & Carcinoma basocelular & Queratosis actínicas & $\begin{array}{c}\text { Carcinoma } \\
\text { escamocelular }\end{array}$ \\
\hline
\end{tabular}




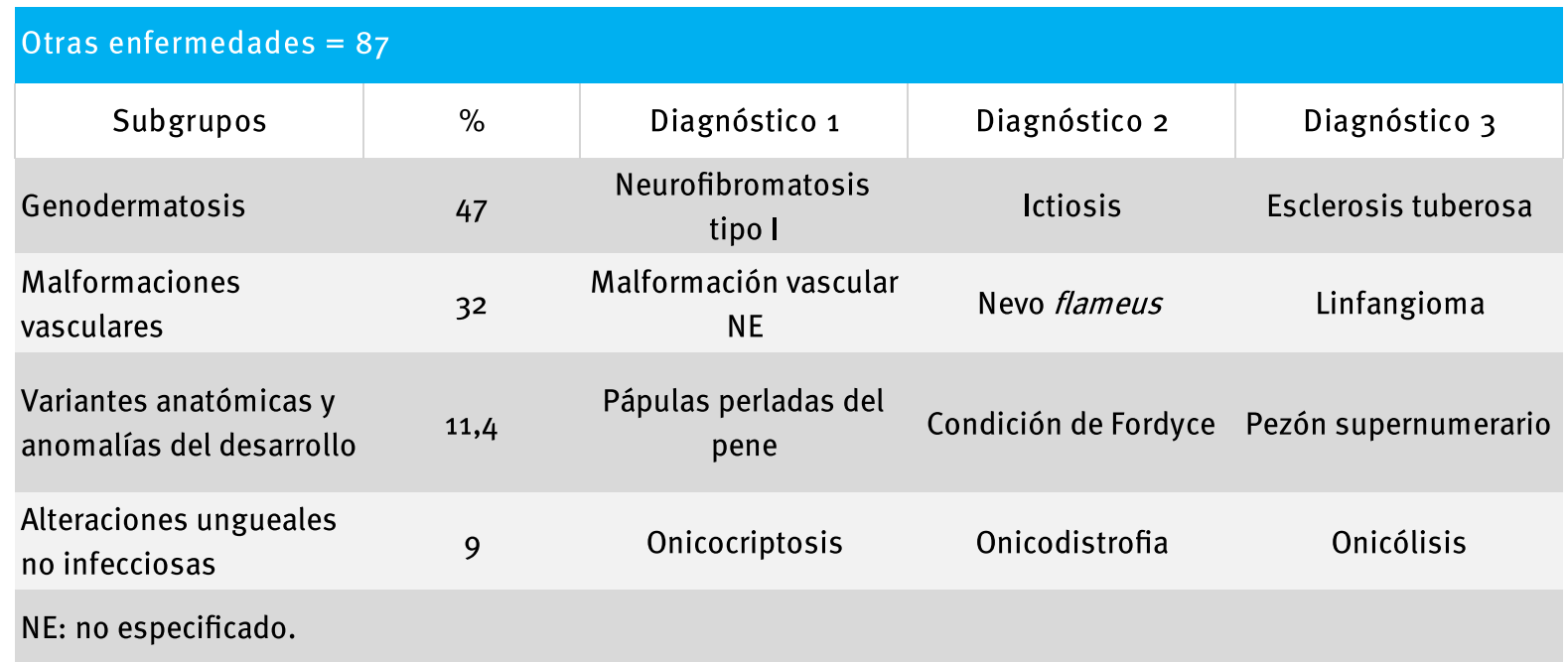

Tabla 4. . Concordancia entre el médico general y el dermatólogo por grupos de enfermedades

\begin{tabular}{|c|c|c|c|c|c|c|c|c|}
\hline & & & \multicolumn{5}{|c|}{ Diagnóstico dermatólogo } & \multirow{2}{*}{ Total } \\
\hline & & & Infecciosas & Inflamatorias & Nbenignas & Nmalignas & Otras & \\
\hline \multirow{2}{*}{\multicolumn{2}{|c|}{ Infecciosas }} & $\mathrm{n}$ & 481 & 237 & 41 & 23 & 9 & 791 \\
\hline & & $\%$ & $74,70 \%$ & $12 \%$ & $5,80 \%$ & $4,20 \%$ & $15,30 \%$ & $20,20 \%$ \\
\hline \multirow{8}{*}{$\begin{array}{c}\text { Diagnóstico } \\
\text { médico } \\
\text { general }\end{array}$} & \multirow{2}{*}{ Inflamatorias } & $\mathrm{n}$ & 130 & 1671 & 48 & 57 & 9 & 1915 \\
\hline & & $\%$ & $20,20 \%$ & $85 \%$ & $6,80 \%$ & $10,40 \%$ & $15,30 \%$ & $48,80 \%$ \\
\hline & \multirow{2}{*}{ Nbenignas } & $\mathrm{n}$ & 29 & 34 & 568 & 81 & 23 & 735 \\
\hline & & $\%$ & $4,50 \%$ & $1,70 \%$ & $80,60 \%$ & $14,80 \%$ & $39,00 \%$ & $18,70 \%$ \\
\hline & \multirow{2}{*}{ Nmalignas } & $\mathrm{n}$ & 4 & 19 & 42 & 385 & 3 & 453 \\
\hline & & $\%$ & $0,60 \%$ & $1 \%$ & $6 \%$ & $70,50 \%$ & $5,10 \%$ & $11,60 \%$ \\
\hline & \multirow{2}{*}{ Otras } & $\mathrm{n}$ & 0 & 6 & 6 & 0 & 15 & 27 \\
\hline & & $\%$ & $0 \%$ & $0,30 \%$ & $0,90 \%$ & $0 \%$ & $25,40 \%$ & $0,70 \%$ \\
\hline \multicolumn{2}{|c|}{ Total } & $\mathrm{n}$ & 644 & 1967 & 705 & 546 & 59 & 3921 \\
\hline
\end{tabular}

En la concordancia realizada para la clasificación por subgrupos se sigue conservando la relación estadísticamente significativa ( $p=0,0001)$ entre las categorías y un índice Kappa de o,6.X

La concordancia medida por diagnósticos fue completa en el 42,2\% $(n=1832)$, parcial en el 10,2\% $(n=444)$, ninguna en el 47,4\% ( $n=2057)$, y la concordancia agre- gada fue del $52,4 \%$.

La concordancia agregada por grupos de enfermedades fue del 55,5\% para el grupo de neoplasias malignas, del 55\% para el grupo de enfermedades infecciosas, del 48\% para enfermedades inflamatorias, del $40,2 \%$ para neoplasias benignas y del $22,9 \%$ para otras enfermedades. 


\section{Gráfica 3. Patrón operacional de la concordancia agregada durante el periodo de estudio}

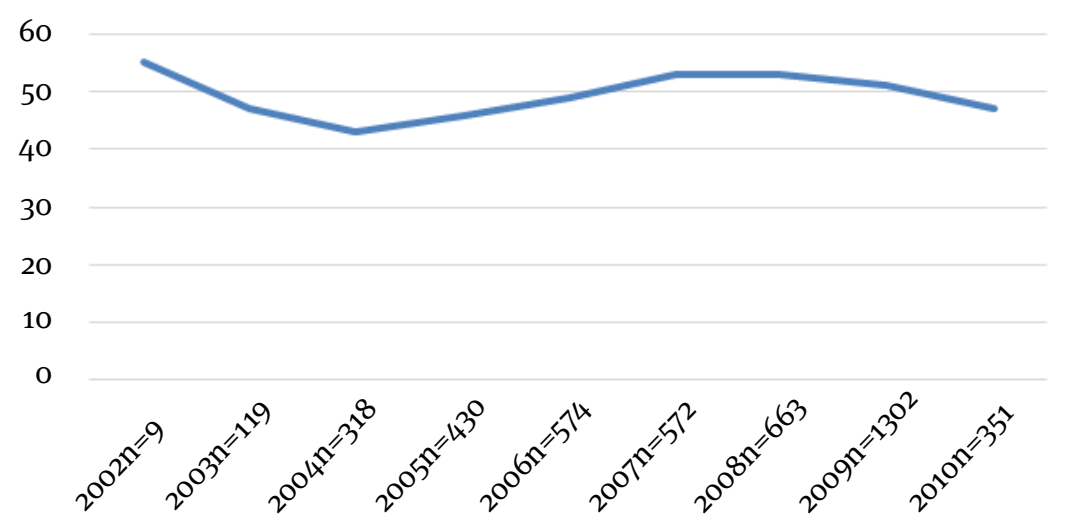

Figura 3. Patrón operacional de la concordancia agregada durante el período de estudio.

En la figura 3 se muestra el patrón operacional de la concordancia agregada durante el período de estudio, la cual muestra poca variabilidad.

La capacidad de resolución mediante la teleconsulta fue del $68,5 \%(n=2971)$, lo que indica como conducta el alta en el $38,7 \%$ de los casos, manejo por el médico general en el $35,7 \%$ y nuevo control por teledermatología en el $16,8 \%$, mientras que en el $22,2 \%(n=964)$ se decidió derivar a la consulta presencial de dermatología en el 95,4\% y de otras especialidades en el 4,5\% (cirugía plástica y genética). En el 12,9\% $(n=560)$ de los casos el médico general realizó la biopsia; en el 70\% de los casos la indicación fue la sospecha de neoplasias malignas. Los motivos de derivación a la consulta presencial se muestran en la figura 4.

Los procedimientos que se solicitaron para la consulta presencial fueron: criocirugía, 56,5\% $(n=195)$; cirugía, $32,7 \%(n=113)$; biopsia, 10,1\% $(n=35)$; infiltración y onicectomía, o,6\% $(n=2)$.

En 94 casos se reportaron dificultades para realizar el diagnóstico. Los motivos encontrados fueron datos insuficientes en la historia clínica y problemas con las imágenes (imágenes desenfocadas), en el 57,7\% y el $42,3 \%$, respectivamente.

\section{DISCUSIÓN}

No queda duda alguna de que la telemedicina es una disciplina en expansión. Debido a la importancia de la imagen, la dermatología es una de las especialidades en las que ha tenido mayor desarrollo y aplicaciones, considerándose como una excelente herramienta complementaria, más no sustitutiva de la consulta presencial dermatológica. En nuestro país, tanto la creciente demanda de la consulta dermatológica como las condiciones geográficas, políticas y sociales hacen necesaria la creación de nuevos métodos de atención en salud, como la telemedicina.

Los programas de telemedicina se vienen desarrollando en diferentes lugares del país desde el 2003 y logran una mayor estabilidad a partir de 2012. Para el 2014, aproximadamente el 15,8\% de los prestadores de salud ofrecieron alguna actividad en teledermatología (9), siendo el programa de Teledermatología de la Universidad de Caldas uno de los pioneros.

Entre las teleconsultas realizadas en el programa, se encontró que solo el 28,6\% pertenece a la edad pediátrica, con una edad media de $37 \mathrm{y}$ un intervalo de consulta más frecuente entre los 18 y los 45 años, similar a lo que se reporta en diferentes estudios, donde el rango de edad oscila entre 1,5 meses y 92 años, con una media entre 37,7 y 45,5 años ${ }^{(4,5,14,15)}$. Esto parece definir un perfil medio de edad de los pacientes que consultan 


\section{Gráfica 4. Motivos de derivación a la consula presencial}

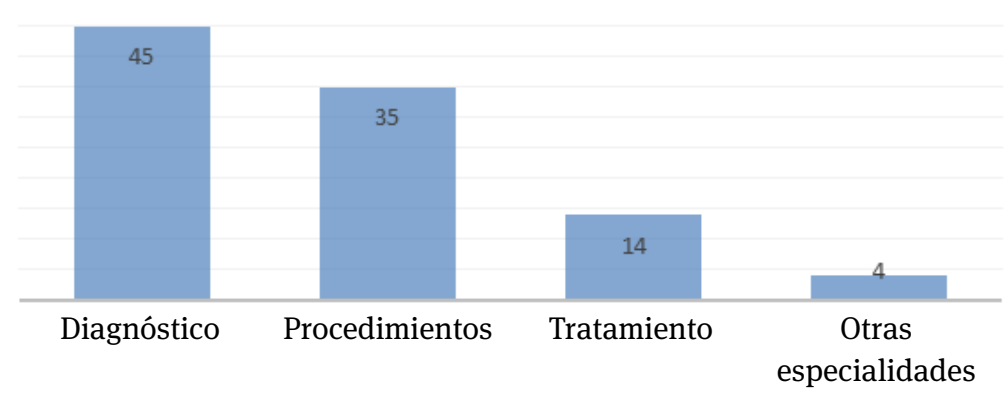

Figura 4. Motivos de derivación a la consulta presencial.

por motivos dermatológicos situado entre la cuarta y la quinta década de la vida.

Tanto en la población pediátrica como en los adultos encontramos un porcentaje de consulta mayor en mujeres, igual que en otros estudios, donde los promedios varían entre el 56,9\% y el 66\% (2, 4, 5, 14-16). Esto podría atribuirse quizá a una mayor preocupación de las mujeres por su aspecto.

Realizar comparaciones entre la frecuencia de las enfermedades consultadas por teledermatología es difícil, ya que hay una amplia variabilidad en las agrupaciones de los diagnósticos utilizadas entre los diferentes estudios; sin embargo, se logra establecer algunos grupos y enfermedades como los motivos de consulta más frecuentes, que fueron muy similares a lo encontrado en este estudio.

Lasierra y colaboradores ${ }^{(17)}$ encontraron que las enfermedades inflamatorias (dermatosis papuloescamosas y eccemas, purpuras y brote, enfermedades vesiculoampollosas) fueron el grupo más común, seguido de los tumores, principalmente los benignos, luego por el grupo de las infecciones y, en última instancia, por el grupo de otras enfermedades en las que se incluyen las genodermatosis, las enfermedades de mucosas, pelo y uñas y las alteraciones vasculares.

Batalla y colaboradores ${ }^{(18)}$ encontraron también que el grupo de enfermedades inflamatorias fue el más fre- cuente, con el $45 \%$ de los casos, seguido de los tumores benignos, con el 16\%, las enfermedades infecciosas, con el 13\%, y los tumores malignos, con el 10\%.

En la población pediátrica se encontraron resultados similares a los de este estudio. Batalla y colaboradores (18) reportaron la patología inflamatoria como la más frecuente en el 39\% de los casos, representada por eccemas principalmente, seguida de las lesiones pigmentadas benignas en el $23 \%$ y la patología infecciosa en el $20 \%$. Igual que en este estudio, no se encontraron consultas en los grupos de tumores malignos. Chen y colaboradores ${ }^{(16)}$ encontraron como los diagnósticos más frecuentes en la edad pediátrica la dermatitis atópica, seguida por los nevos, en el 13,9\%, las verrugas, en el 10,4\% de los casos, y el molusco contagioso, en el $9,7 \%$ de los casos.

La concordancia diagnóstica es uno de los puntos más evaluados entre los diferentes estudios en teledermatología. La concordancia que más se describe es entre dermatólogos de la teleconsulta y la consulta presencial, la cual muestra valores que oscilan entre el $87,8 \%$ y el $90 \%{ }^{(13,18,19)}$, que es ligeramente mejor para patología infecciosa $(91,3 \%)$, inflamatoria $(86,4 \%)$ y tumoral $(87,2 \%)^{(19)}$.

La concordancia entre el pediatra y el dermatólogo se describe solo en un estudio. Esta fue completa en el $55 \%$ y parcial en el $10 \%{ }^{(2)}$. 


\section{Puntos clave}

- Teledermatología una disciplina en expansión

- Programa de Teledermatologia de la Universidad de Caldas pionero en Colombia, una herramienta fundamental en la educación médica

- En la población estudiada fueron más frecuentes las teleconsultas del sexo femenino, con edades entre la cuarta y quinta década de la vida, por dermatosis clasificadas en enfermedades inflamatorias.

La concordancia entre el médico general y el dermatólogo encontrada en este estudio fue completa en el $46,6 \%$ y agregada en el 55,3\%, similar a lo reportado en

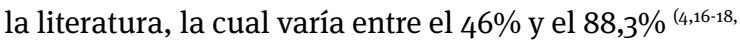
20, 21), considerándose en general como buena e incluso siendo comparable con la del pediatra. Encontramos que la concordancia agregada fue mejor para el grupo de neoplasias malignas, seguida por las enfermedades infecciosas y las enfermedades inflamatorias, semejante a lo reportado en otro estudio donde se describe mejor para enfermedades infecciosas $(62 \%)$ e inflamatorias $(55 \%)^{(17)}$.

En la medición de la concordancia en función de los diagnósticos clasificados por grupos se encontró una asociación y una concordancia adecuada para la mayoría de los grupos de enfermedades (inflamatorias, $85 \%$; neoplasias benignas, 80,6\%; infecciosas, $74,7 \%$; neoplasias malignas, 70,5\%; y otras enfermedades, 25,4\%); sin embargo, cuando la asociación se realiza con agrupaciones más cercanas a los diagnósticos (subgrupos), el valor de concordancia disminuye. Esto puede deberse a que el diagnóstico del médico general y del dermatólogo fueron diferentes, pero los diagnósticos pertenecían al mismo grupo de enfermedades, $\mathrm{y}$ también a la utilización de términos generales y no de diagnósticos más específicos.

En la literatura se habla de la mejoría en la concordancia y en las habilidades de los médicos generales con el uso de la teledermatología ${ }^{(17,18,21)}$, con una disminución significativa en el número de teleconsultas durante el primer año ${ }^{(22,23)}$. Consideramos que el hecho de pertenecer a un programa de teledermatología le facilita al médico general obtener formación continua para aumentar su capacidad diagnóstica a través de la retroalimentación, con una mejoría en la selección de los pacientes y una disminución en la derivación de estos. Según otros estudios, la mejoría puede reflejarse también tras la aplicación de protocolos para la selección de los pacientes ${ }^{(22,23)}$.
Los médicos generales encuestados en dos estudios declararon que aprendieron de la respuesta del dermatólogo en el $85 \%$ de todas las teleconsultas ${ }^{(22)}$. En otro estudio señalaron que el $58 \%$ aprendió del plan de tratamiento y el $63 \%$ del diagnóstico debido a una mejor comunicación con el dermatólogo ${ }^{(24)}$.

En este estudio se encontró una concordancia agregada, que se mantuvo relativamente estable durante el tiempo, lo que muestra que los médicos remisores tenían un nivel de conocimientos en dermatología que fue similar entre ellos; sin embargo, no se observó mejoría de esta a través de los años. Esto puede ser explicado por la rotación constante de los médicos generales o rurales que trabajan en los diferentes municipios, por lo cual puede que no haya un continuo en la retroalimentación.

La capacidad de resolución fue alta y concuerda con lo publicado por otros autores ${ }^{(17,19,22)}$. Se describe más en el grupo de enfermedades inflamatorias $(62,8 \%)$, lesiones melanocíticas $(59,2 \%)$ y tumores benignos $(55,2 \%)^{(18)}$. Como es de esperarse, ante la sospecha de malignidad, el porcentaje de derivación encontrado en otros estudios es del $96,7 \%{ }^{(18)}$. En el análisis de los casos de sospecha de malignidad cabe recalcar la importancia de la dermatoscopia; en un estudio realizado por Massone y colaboradores ${ }^{(25)}$, los autores encontraron que, en teledermatología, la combinación de imágenes clínicas y dermatoscópicas usadas con el objetivo de establecer un sistema de filtro para el cáncer de piel lograron evitar el $82 \%$ de las derivaciones. En el programa no se incluyeron imágenes de dermatoscopia, consideramos que sería lo ideal; sin embargo, puede ser difícil de aplicar en una entidad de primer nivel, ya que requiere de mayor costo en equipos (dermatoscopio) y capacitación.

El estándar de oro en el diagnóstico del cáncer de piel sigue siendo la histología. La teleconsulta tiene la ventaja de permitir dar instrucciones al médico general 
para la toma y el envío del espécimen de biopsia desde el centro periférico, para posteriormente dar el diagnóstico y la programación quirúrgica a distancia. En el programa de Teledermatología, el médico general tomó las biopsias en 560 pacientes, de las cuales, el $70 \%$ fue por sospecha de neoplasias malignas. En este contexto, el paciente debe desplazarse a la consulta presencial una vez para su tratamiento definitivo, para disminuir los costos relacionados con la movilización y la baja laboral del paciente y su acompañante.

Los motivos de derivación a la consulta presencial encontrados son similares a los de la literatura; principalmente fueron la necesidad de confirmación diagnóstica y los procedimientos como crioterapia y biopsias ${ }^{(2,18,19)}$.

En cuanto a las dificultades al momento de emitir el diagnóstico en la teleconsulta, se encontró que en el $42,3 \%$ estas fueron por imágenes desenfocadas. El hecho de que el abordaje diagnóstico de las lesiones dermatológicas se haga principalmente a través de la vista explica por qué se enfatiza que la adecuada adquisición de las imágenes representa el aspecto más crítico de la interconsulta dermatológica a distancia.

Otro de los enormes beneficios que presenta el programa es la educación médica continuada, dirigida a la formación de estudiantes de pregrado y posgrado. Durante el período de funcionamiento del programa se acumuló un material considerable para la docencia y la academia, ya que la plataforma cuenta con más de 5000 casos y cerca de 20.000 fotos clínicas, lo que permite hacer consulta docente-asistencial sin limitación de casos clínicos, ni restricción en el número de estudiantes. Shaikh y colaboradores (26) aplicaron la teledermatología en el aprendizaje de 44 estudiantes entre residentes de pediatría y estudiantes de tercer año de Medicina. Encontraron un 22\% de mejoría en 5 áreas de las 6 evaluadas: diagnóstico, 26\%; tratamiento, $16 \%$; examen físico, $21 \%$; conocimiento, $41 \%$; y diagnóstico diferencial, $21 \%$. Lo que afirma la importancia del proceso de retroalimentación como método de aprendizaje.

\section{CONCLUSIÓN}

En conclusión, se muestra una casuística significativa de la experiencia obtenida durante el período de servicio del programa de Teledermatología de la Universidad de Caldas.

Esto permite definir varios puntos: las características sociodemográficas y las enfermedades consultadas en la población Caldense presentan una distribución similar a la reportada en otros estudios. El grado de concordancia del diagnóstico entre el médico general y el dermatólogo es adecuado; de forma general se puede concluir que el médico general es capaz de clasificar la enfermedad por grandes grupos, pero puede fallar al requerir una precisión en el diagnóstico. Por otra parte, se demuestra que la capacidad resolutiva del programa es alta y logra evitar más de la mitad de las derivaciones a la consulta presencial. Se adquiere una perspectiva de lo que enfrenta el médico general y de las enfermedades que puede manejar mediante la teleorientación, lo que impacta en la disminución de los costos para el paciente y su acompañante. Pocos casos tuvieron inconvenientes al emitir un diagnóstico y fueron susceptibles de mejorar mediante la implementación estricta de protocolos de fotografía dermatológica. Se muestra cómo estos programas se convierten en una herramienta básica y fundamental para la educación de los estudiantes de pregrado y posgrado, el médico general y los especialistas. Finalmente, se busca motivar la formación de nuevos centros de teledermatología en el país para mejorar la cobertura y la oportunidad de atención de los pacientes con enfermedades de la piel.

\section{AGRADECIMIENTOS}

Especial agradecimiento al Dr. Juan Manuel Pérez por su aporte en el análisis estadístico.

\section{REFERENCIAS}

1. Martínez-García S, del Boz-González J, MartínGonzález T, Samaniego-González E, Crespo-Erchiga V. [Teledermatology. Review of 917 teleconsults]. Actas Dermosifiliogr. 2007;98(5):318-24.

2. Batalla A, Suh-Oh HJ, Abalde T, Salgado-Boquete L, de la Torre C. [Teledermatology in Paediatrics. Observations in daily clinical practice]. An Pediatr (Barc). 2016;84(6):324-30. doi: 10.1016/j.anpedi.2015.07.005

3. Armstrong AW, Wu J, Kovarik CL, Goldyne ME, Oh $\mathrm{DH}, \mathrm{McKoy} \mathrm{KC}$, et al. State of teledermatology programs in the United States. J Am Acad Dermatol. 2012;67(5):939-44. doi: 10.1016/j.jaad.2012.02.019

4. Figueroa A, Barrios X, Radrigan C, Valdés P, Zemelman V. Aplicación de la teledermatología asincrónica en usuarios de la comuna de Yerba Buenas, Región del Maule, Chile. Rev Chilena Dermatol. 2011;27(2):170-6.

5. Castillo-Arenas E, Garrido V, Serrano-Ortega S.Skin conditions in primary care: an analysis of referral 
demand. Actas Dermosifiliogr. 2014;105(3):271-5. doi: 10.1016/j.adengl.2013.10.005

6. Avellaneda C, Seidel A, Londoño A, González C. Caracterización socioeconómica, geográfica y laboral de los médicos dermatólogos en Colombia. Rev Asoc Colomb Dermatol. 2012;20(2):129-34.

7. Resolución 2003 de 2014. Por la cual se definen los procedimientos y condiciones de inscripción de los Prestadores de Servicios de Salud y de habilitación de servicios de salud. Diario Oficial n.o 49.167. Disponible en: https://bit.ly/3pKQbXS

8. Ley 1341de 2009. Por la cual se definen principios y conceptos sobre la sociedad de la información y la organización de las tecnologías de la información y las comunicaciones -TIC- se crea la agencia nacional de espectro y se dictan otras disposiciones. Diario Oficial n.o 47.426. Disponible en: https:// bit.ly/33i2SzZ

9. Línea de base de telemedicina en municipios priorizados - Colombia. Resultados Generales [Internet]. Ministerio de Salud y Protección Social; 2015. Disponible en: https://bit.ly/2IW29g9

10. Telesalud [internet]. Universidad de Caldas [2015]. Disponible en: http://telesalud.ucaldas.edu.co/ telesalud/

11. Mora O, Rodríguez N, Torres M, Rochel C, Olmos E. Acuerdo entre el examen dermatológico directo y teledermatología asincrónica prueba piloto, Hospital de San José, Bogotá DC, Colombia. Repert Med Cir. 2012;21(2):122-25. doi: 10.31260/RepertMedCir.v21.n2.2012.806

12. Ruiz C, Gaviria C, Gaitán M, Manrique R, Zuluaga A, Trujillo A. Concordance studies of a web based system in teledermatology. Colomb Med. 2009;40(3):259-70.

13. Jaramillo F, Santacoloma G, Montoya J, Arbeláez M. Concordancia entre el diagnóstico presencial y telediagnóstico en pacientes dermatológicos. Acta Med Colomb. 2003;28(1):11-4.

14. Sanclemente G, Mahecha M, Guzmán C. Enfermedades de la piel más frecuentes en la consulta externa dermatológica del Hospital Universitario San Vicente de Paul y del Hospital Infantil, 1999. Acta Med Colomb. 2001;26(5):240-4.

15. Gatica J, Bertoló S, Morales E, Espinoza M, Contreras C. Teledermatología en Chile, un aporte a la atención primaria de salud. Piel. 2015;30(3):14854 .

16. Chen TS, Goldyne ME, Mathes EF, Frieden IJ, Gilliam AE. Pediatric teledermatology: observations based on 429 consults. J Am Acad Dermatol. 2010;62(1):61-6. doi: 10.1016/j.jaad.2009.05.039
17. Lasierra N, Alesaco A, Gilaberte Y, Magallón R, García J. Lessons learned after a three-year store and forward teledermatology experience using internet: Strengths and limitations. Int J Med Inform. 2012;81(5):232-43. doi: 10.1016/j.ijmedinf.2012.02.008

18. Batalla A, Suh-Oh H, Salgado L, Abalde T, de la Torre C. Teledermatología. Capacidad para reducir consultas presenciales según el grupo de enfermedad. Piel. 2015;31(3):156-63. doi: 10.1016/j. piel.2015.09.013

19. Vañó-Galván S, Hidalgo A, Aguayo-Leiva I, GilMosquera M, Ríos-Buceta L, Plana MN, et al. [Store-and-forward teledermatology: assessment of validity in a series of 2000 observations]. Actas Dermosifiliogr. 2011;102(4):277-83. doi: 10.1016/j. ad.2010.11.006

20. Oakley A, Reeves F, Bennett J, Holmes S, Wickham H. Diagnostic value of written referral and/ or images for skin lesions. J Telemed Telecare. 2006;12(3):151-8. doi: 10.1258/135763306776738620

21. Mesa J, Jaramillo F, Benjumea M. Factores relacionados con la consulta tardía a dermatología por cáncer de piel. prueba piloto. departamento de Caldas. Hacia la Promoción de la Salud. 2008;13:178-94.

22. van der Heijden J, de Keizer NF, Bos JD, Spuls PI, Witkamp L. Teledermatology applied following patient selection by general practitioners in daily practice improves efficiency and quality of care at lower cost. Br J Dermatol. 2011;165(5):1058-64. doi: 10.1111/j.1365-2133.2011.10509.x

23. Landow SM, Mateus A, Korgavkar K, Nightingale D, Weinstock MA. Teledermatology: key factors associated with reducing face-to-face dermatology visits. J Am Acad Dermatol. 2014;71(3):570-6. doi: 10.1016/j.jaad.2014.02.021

24. van der Akker T, Reker C, Knol A, Post J, Wilbrink J, van der Veen J. Teledermatology as a tool for communication between general practitioners and dermatologists. J Telemed Telecare. 2001;7(4):193-8. doi: 10.1258/1357633011936390

25. Massone C, Maak D, Hofmann-Wellenhof R, Soyer HP, Fruhauf J. Teledermatology for skin cancer prevention: an experience on 690 Austrian patients. J Eur Acad Dermatol Venereol. 2014;28(8):1103-8. doi: $10.1111 /$ jdv.12351

26. Shaikh N, Lehmann CU, Kaleida PH, Cohen BA. Efficacy and feasibility of teledermatology for paediatric medical education. J Telemed Telecare. 2008;14(4):204-7. doi: 10.1258/jtt.2008.071108 